\title{
Far-infrared absorption in GaAs:Te Liquid Phase Epitaxial films
}

\author{
B L Cardozo and E E Haller \\ Department of Materials Science and Engineering, University of California, Berkeley, \\ 94720 and
}

Materials Sciences Division, Lawrence Berkeley National Laboratory, Berkeley, California, 94720

L A Reichertz

Department of Physics, University of California, Berkeley, California, 94720

\begin{abstract}
J W Beeman
Materials Sciences Division, Lawrence Berkeley National Laboratory, Berkeley, California, 94720
\end{abstract}

The far-infrared absorption spectrum of n-type GaAs is of interest for applications such as GaAs photoconductors and Blocked Impurity Band (BIB) detectors. The linear optical absorption coefficients $\alpha$ for three n-type GaAs films of varying doping concentrations have been measured in the range of 10 to 100 $\mathrm{cm}^{-1}$ using Fourier Transform Infrared Spectrometry. These results show $\alpha$ having maximum values of between $46 \mathrm{~cm}^{-1}$ at $1 \times 10^{15} \mathrm{~cm}^{-3}$ and approximately $800 \mathrm{~cm}^{-1}$ at $2.1 \times 10^{16} \mathrm{~cm}^{-3}$. The formation and widening of a donor impurity band with increasing impurity concentration is clearly demonstrated. 


\subsection{Fd, 78.30.Fs, 78.20.Ci, 73.20.Hb}

GaAs is an attractive semiconductor for photoconductor based optical detectors (Blocked Impurity Band detectors, or $\left.\mathrm{BIBs}^{1}\right)$ in the far-infrared due to its low donor electron binding energy $(6 \mathrm{meV}){ }^{2}$ This property makes extrinsic GaAs sensitive to radiation of wavelengths greater than $300 \mu \mathrm{m}$, while current photoconductor technology operates only up to $220 \mu \mathrm{m}$. Photoconductor detectors are highly desirable for application in high-altitude and space-based far-infrared telescopes due to their high sensitivity and ease of integration compared to thermal detectors (bolometers). Efforts to develop such devices are underway, ${ }^{3}$ however, their design requires a knowledge of the optical absorption characteristics of n-type doped GaAs in the far-infrared. A determination of the broadening of the donor impurity band in GaAs with increasing dopant concentration is also required, as this effect leads to a reduction of the shallow donor thermal ionization energy and longer wavelength response. The low temperature far-infrared absorption spectrum of n-type GaAs has not been measured quantitatively as a function of doping concentration.

Here we present a study based on absorption spectrometry to determine the far-infrared linear optical absorption coefficients for n-type GaAs doped between $1 \times 10^{15} \mathrm{~cm}^{-3}$ and $2.1 \times 10^{16} \mathrm{~cm}^{-3}$. Previous studies of GaAs donor absorption and photoconductivity ${ }^{4,5,6}$ have not focused on attaining the linear optical absorption coefficient as a function of doping. Far-infrared absorption studies performed by Summers et al. ${ }^{3}$ revealed an absorption maximum at $37 \mathrm{~cm}^{-1}$ for lightly doped samples, in agreement with our results, but did not quantitatively determine the shallow donor absorption coefficients. Bosomworth et al. ${ }^{4}$ determined $\alpha$ only for one doping concentration $\left(1 \times 10^{15} \mathrm{~cm}^{-3}\right)$.

Four GaAs epitaxial films containing different concentrations of the group VI donor dopant Te were grown via Liquid Phase Epitaxy. Growth was performed in a single-crystal sapphire crucible, using a semi-insulating (100) GaAs substrate and high-purity (8-9s) Ga solvent. Semi insulating GaAs was added into the Ga solvent as the arsenic source for film growth. An appropriate quantity of heavily doped GaAs:Te was added to introduce the dopant. Te was chosen as the dopant because of its low diffusivity in the solid phase ${ }^{7}$ and its relatively low vapor pressure ${ }^{8}$. The crucible was contained inside a silica process tube with Pd-diffused flowing hydrogen gas at 1 atmosphere. The entire system was brought to $800^{\circ} \mathrm{C}$ and left for four hours to allow the GaAs source material to dissolve and saturate the Ga solvent. Growth was initiated by tipping the $\mathrm{Ga}+\mathrm{As}+\mathrm{Te}$ solution onto the GaAs substrate and lowering the temperature of the 
system by $0.5^{\circ} \mathrm{C} / \mathrm{min}$. The resulting Te concentrations in the films vary from $1 \times 10^{15} \mathrm{~cm}^{-3}$ to $2.1 \times 10^{16} \mathrm{~cm}^{-3}$ (Table I). Based on the measured free electron concentration and mobility we estimate the compensation ${ }^{9}$ of the films to be between $60-70 \%$ for samples 294 and 295 , and $80-90 \%$ for sample 286 .

All samples were lapped and polished before analysis. Lapping using .3 $\mu \mathrm{m}$ alumina grit slurry was followed by colloidal silica chemo-mechanical polishing on a rotary polishing machine. Lapping was performed to adjust the total absorbance ( $\alpha$ x) of each sample in order to optimize the sensitivity of absorption measurements and to achieve as flat a film surface as possible. The substrate face of each sample was lapped at a $1^{\circ}$ angle towards the epilayer to avoid Fabry-Perot oscillations within the sample. Absorption measurements were taken at $1.35 \mathrm{~K}$ in a pumped liquid He test Dewar, using a stepping mirror Fourier transform infrared spectrometer with a $50 \mu \mathrm{m}$ thick Mylar beamsplitter. Cold black polyethylene and a cold $100 \mathrm{~cm}^{-1}$ low pass filter ${ }^{10}$ were inserted into the beam path to reject band-edge light and to increase the signal to noise ratio in the spectral band of interest. Transmission was measured using a neutron transmutation doped (NTD) Ge bolometer detector. ${ }^{11}$ The light source, a mercury arc lamp, was chopped at $13 \mathrm{~Hz}$.

The substrate absorption was cancelled by taking a ratio of the total transmitted intensities of the film+substrate to that of a semi-insulating GaAs reference substrate of the same wedged configuration, $I_{\text {film }+ \text { sub }} / I_{\text {sub }}$. The relation of the absorption coefficient to the transmission signal and sample thickness is given by Equation 1.

$\frac{I_{\text {film }+ \text { sub }}}{I_{\text {sub }}}=\frac{(1-R)^{2} e^{-\alpha_{\text {film }} t_{\text {film }}}}{1-R^{2} e^{-2 \alpha_{\text {film }} t_{\text {film }}}}$

Here $\alpha$ is the linear optical absorption coefficient, $\mathrm{R}$ is the reflectivity, and $\mathrm{t}$ is thickness. The equation takes into account the multiple internal reflections that can occur within the sample and substrate. We have assumed the absorption coefficient of the semi-insulating substrate to be negligibly small. This assumption is justified by the low extinction coefficient of the material in the range of 10 to $100 \mathrm{~cm}^{-1}\left(1.2 \text { to } 3.6 \times 10^{-3}\right)^{12}$ giving rise to an absorption coefficient of $\alpha=.34 \mathrm{~cm}^{-1}$. Such a small value can be neglected compared to the absorption of the doped films. The reflectivity is estimated to be $\mathrm{R}=0.317$ based on measurements of the index of refraction for semi-insulating GaAs in the frequency range of interest. ${ }^{8}$ The absorption spectra obtained from the three films after performing a ratio with the reference sample are shown in Figure 1 at a 
resolution of $2 \mathrm{~cm}^{-1}$. For comparison the photoconductivity spectrum of a $1 \times 10^{14} \mathrm{~cm}^{-3}$ unintentionally doped n-type film (sample 191) is also shown in Figure 1. The sharp photoconductivity peak at $35.5 \mathrm{~cm}^{-1}$ is due to the $1 \mathrm{~s}-2 \mathrm{p}$ bound excited state transition followed by thermal ionization or tunneling into the conduction band. A second, broader peak at $49 \mathrm{~cm}^{-1}$ represents 1 s-continuum transitions. The deliberately Te doped samples 286, 294 and 295 show absorption maxima near $37 \mathrm{~cm}^{-1}$, between the $1 \mathrm{~s}-2 \mathrm{p}$ and $1 \mathrm{~s}-$ continuum transition energies, and represent a combination of both types of excitation.

In Figure 2 the absorption coefficients at several different wavenumbers are compared. Error bars have been included to account for the variation in thickness between the center and edges of the sample. The scaling of the absorption coefficient with the doping concentration is linear as expected. Far-infrared absorbance is demonstrated at longer wavelengths compared to the photoconductive response of the high purity GaAs. It is this extended wavelength response which makes GaAs highly desirable as a material for far-infrared detector production. Furthermore, as doping is increased the spectra become broader. The formation and broadening of the impurity band is due largely to two factors. First, the decreased spacing forces Te donor wavefunctions to overlap spatially, leading to an exchange interaction and dispersion of the associated energy eigenstates. At a high enough concentration, a band of energy states (in which the donor electrons are delocalized) forms within the GaAs forbidden gap. The transition from an insulating conduction state to a metallic one represents the metal-insulator transition. ${ }^{13}$ The doping concentration at which the metal-insulator transition occurs can be estimated by the Mott condition, $\mathrm{N}^{1 / 3} \mathrm{a}_{\mathrm{h}}=.25$, where $\mathrm{N}$ is the shallow impurity concentration and $\mathrm{a}_{\mathrm{h}}$ is the corresponding Bohr radius. For shallow donors in GaAs, the Mott transition occurs at approximately $2 \times 10^{16} \mathrm{~cm}^{-3}$. At still higher doping, the impurity band will eventually merge with the conduction band, eliminating the optical excitation path of shallow donor electrons to the conduction band. Second, broadening occurs due to Coulombic interaction between neutral and ionized impurities. The relative importance of this effect depends upon the compensation level of the material. ${ }^{14}$ The broadening due to the exchange interaction can be estimated using a simplified model of donors occupying a simple cubic lattice. Broadening due to exchange interaction for a donor electron is related to the overlap integral with its nearest neighbors (coordination number $\mathrm{z}=6$ for simple cubic) $\mathrm{B}=2 \mathrm{zI}$. The overlap integral $\mathrm{I}=\int \Psi(|r-a|) H \Psi(r) d r \approx 5 \mathrm{E}_{\mathrm{ry}} \exp \left(\mathrm{a} / \mathrm{a}_{\mathrm{h}}\right)$, where $\mathrm{E}_{\mathrm{ry}}$ is the Rydberg energy $(\sim 4.6$ $\mathrm{meV}$ ), $\mathrm{a}$ is the average inter-impurity spacing (taken to be $\mathrm{N}_{\mathrm{d}}^{-1 / 3}$ ), and $\mathrm{a}_{\mathrm{h}}$ is the Bohr radius for donors in 
GaAs. Upon applying this theory (Table I) to the three samples we find relatively good agreement only at the higher concentrations. The reason for the large underestimation of the broadening at $1 \times 10^{15} \mathrm{~cm}^{-3}$ is most likely due to Stark broadening as discussed above. At the higher concentrations, impurity band broadening has occurred to a much more significant extent, and begins to outweigh the effect of the random electric field dispersion.

The formation and widening of impurity bands is best demonstrated in Figure 3, which displays the spectra for samples 294, 295, and 286 normalized to their respective maxima. For the least doped sample, 286, the absorption spectrum is less broad and slightly shifted to higher energy, as the impurity band in this sample is not well formed at this concentration. The extended absorption at longer wavelength in the more highly doped films is obvious. Stillman et al. ${ }^{15}$ demonstrated this trend in GaAs at lower doping concentrations using photoconductivity measurements. Such measurements are impossible at the doping levels used in this study, since the hopping and impurity band conduction are dominant, exceeding the photocurrent. The data near $70 \mathrm{~cm}^{-1}$ have been omitted in the spectra because they coincide with the first spectral minimum of the beamsplitter, leading to a dramatic reduction of the signal to noise ratio.

In conclusion we have determined the far-infrared linear absorption spectra for compensated, $\mathrm{n}$ GaAs samples doped with Te to between $1 \times 10^{15} \mathrm{~cm}^{-3}$ and $2.1 \times 10^{16} \mathrm{~cm}^{-3}$. The absorption spectra clearly broaden with increasing doping concentration, demonstrating the effects of Stark broadening and of impurity band formation. The shape of the spectra reflects $1 \mathrm{~s}-2 \mathrm{p}$ and $1 \mathrm{~s}-\mathrm{continuum}$ transitions.

This work was supported by NASA Ames Research Center, Order No. A53228D through the U.S. Department of Energy under Contract No. DE-AC03-76SF00098.

\footnotetext{
${ }^{1}$ M D Petroff and M G Stapelbroek, Conf. on Extrinsic Si Detect. Behavior, NOSC, San Diego, CA, (1980).
} 
2 P Y Yu and M Cardona, Fundamentals of Semiconductors, 3rd edition (Springer, Berlin, 2001), p.169.

${ }^{3}$ B L Cardozo, J W Beeman, and E E Haller, Far-IR, Sub-mm \&MM Detector Tech. Wkshp, Monterey, CA, unpublished (2002).

4 C J Summers, R Dingle, and D E Hill, Phys. Rev. 1, 1603 (1970).

5 D R Bosomworth, R S Crandall, and R E Enstrom, Physics Letters 28A, 320 (1968).

${ }^{6}$ C M Wolfe and G E Stillman, Third Intl. Symp. on GaAs and Related Compounds, Aachen, 3, (1971).

${ }^{7}$ M B Dutt and B L Sharma, Diffusion in Compound Semiconductors, Landolt-Börnstein Series (Springer, Heidelberg, 1998), Vol. 33-A, p.3-11.

${ }^{8}$ TRCVP, Vapor Pressure Database, Version 2.2P, Thermodynamic Research Center, Texas A\&M University, College Station, TX.

9 W Walukiewicz, L Lagowski, L Jastrzebski, M Lichtensteiger, and H C Gatos, J. Appl. Phys. 50, 899 (1979).

${ }^{10}$ Supplied by Prof. P. Ade, Cardiff University, UK

11 E E Haller, Fourth Intl. Conf. Neutron Transmutation Doping of Semicond. Matrls., Gaithersburg, MD, 21, (1982).

12 E D Palik, Handbook of Optical Constants of Solids (Academic Press, 1985), p.429.

${ }^{13}$ For further information see B I Shlovskii and A L Efros, Electronic Properties of Doped Semiconducotrs, Springer, 1984

14 D M Larsen, Phys. Rev. B 13, 1681 (1976). 
${ }^{15}$ G E Stillman, C M Wolfe, and J O Dimmock, Third Int. Conf. Photocond., Stanford, CA, 265, (1969). 


\begin{tabular}{|c|c|c|c|c|c|}
\hline Film & $\begin{array}{c}\text { Thickness } \\
(\mu \mathrm{m})\end{array}$ & $\begin{array}{l}\mathrm{N}_{300 \mathrm{~K}} \\
\left(\mathrm{~cm}^{-3}\right)\end{array}$ & $\begin{array}{c}\mu_{300 \mathrm{~K}} \\
\left(\mathrm{~cm}^{2} / \mathrm{Vs}\right)\end{array}$ & $\begin{array}{c}\text { FWHM } \\
\left(\mathrm{cm}^{-1}\right)\end{array}$ & $\begin{array}{c}\text { Theory FWHM } \\
\left(\mathrm{cm}^{-1}\right)\end{array}$ \\
\hline 286 & 40 & $1 \times 10^{15}$ & 5680 & 13 & .15 \\
\hline 294 & 66 & $2.1 \times 10^{16}$ & 4490 & 55 & 68 \\
\hline 295 & 62 & $6.7 \times 10^{15}$ & 5280 & 28 & 13 \\
\hline 191 & 100 & $1.0 \times 10^{14}$ & 7440 & N/A & $\mathrm{N} / \mathrm{A}$ \\
\hline Ref. & $\mathrm{N} / \mathrm{A}$ & SI & $\mathrm{N} / \mathrm{A}$ & $\mathrm{N} / \mathrm{A}$ & $\mathrm{N} / \mathrm{A}$ \\
\hline
\end{tabular}

Table I. samples used in absorption study 
Figure 1. Absorption Spectra for samples $294\left(2.1 \times 10^{16} \mathrm{~cm}^{-3}\right), 295\left(6.7 \times 10^{15} \mathrm{~cm}^{-3}\right)$, and $286\left(1 \times 10^{15} \mathrm{~cm}^{-3}\right)$, and the photoconductivity spectrum for sample $191\left(1 \times 10^{14}\right.$ $\mathrm{cm}^{-3}$ ). The dashed line indicates loss of data due to beamsplitter minimum.

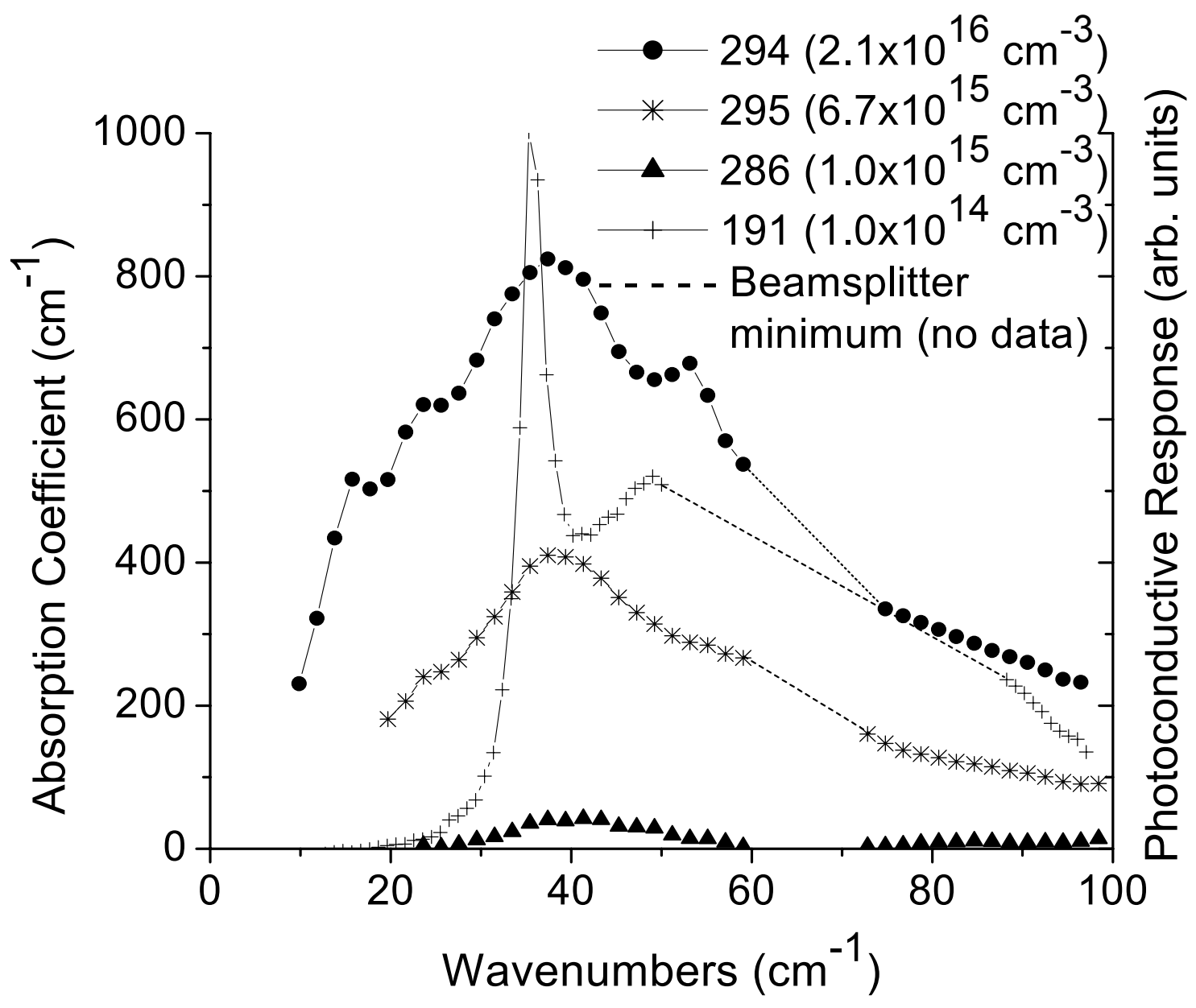


Figure 2. Absorption coefficient versus Te concentration, shows the expected linear relationship.

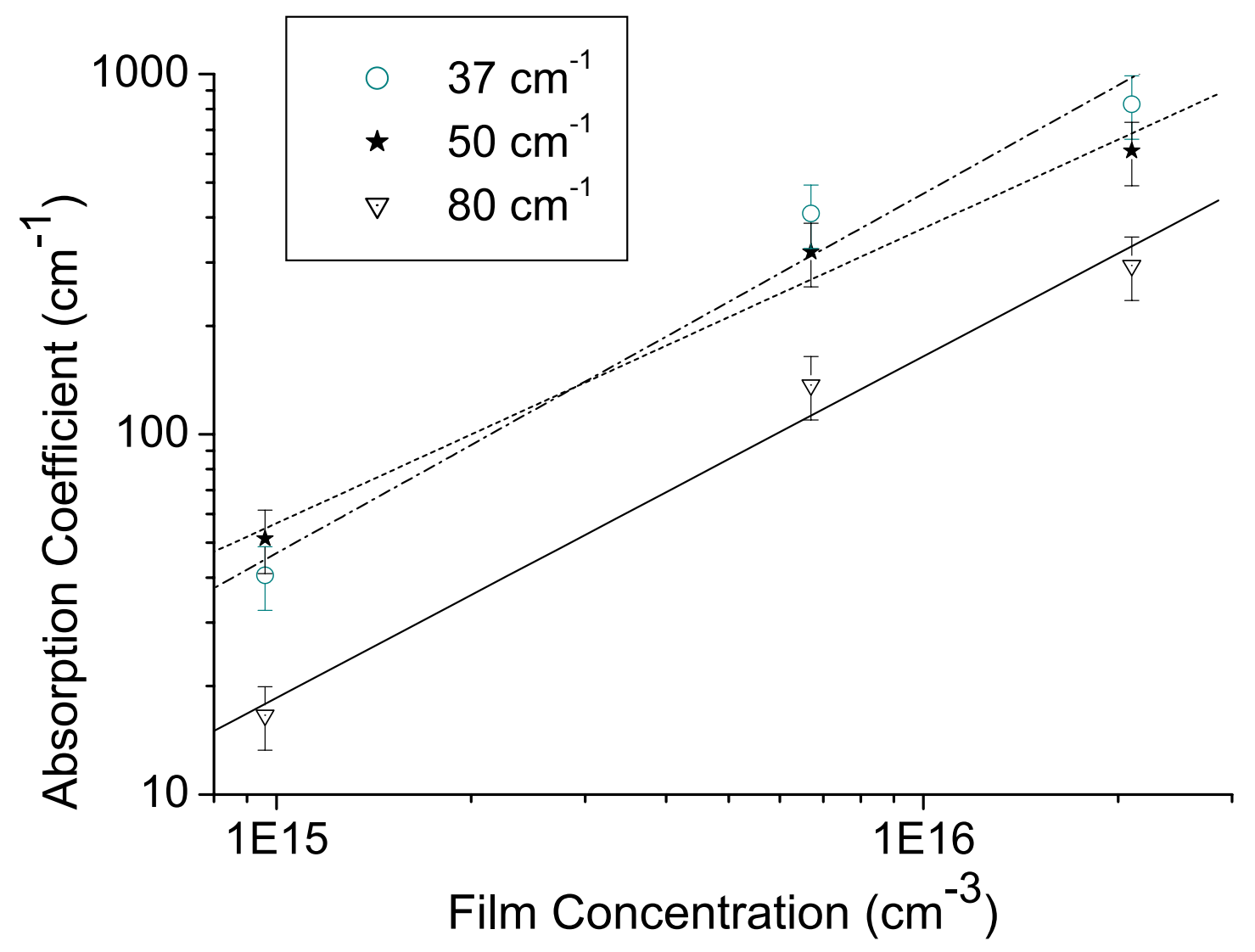




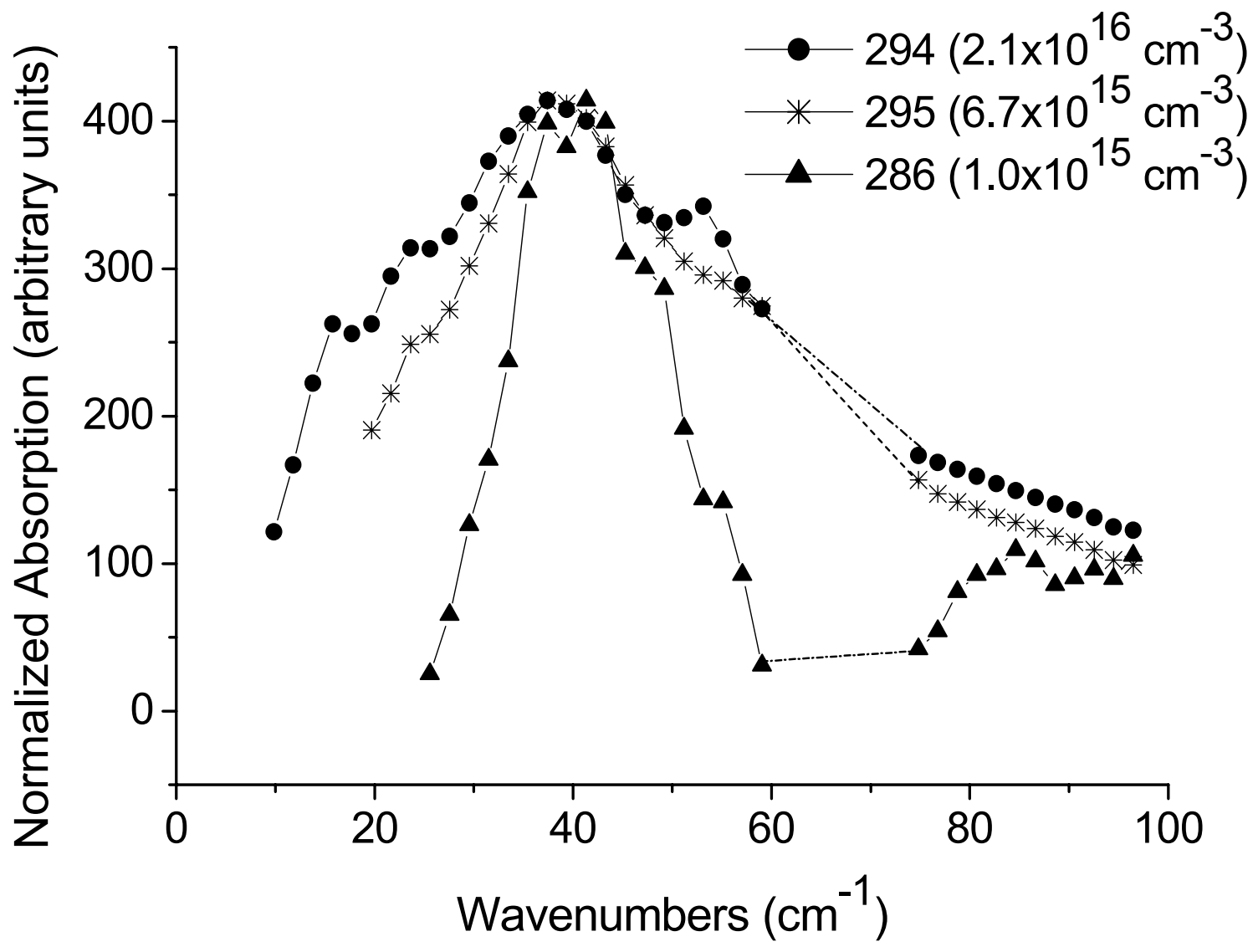

Figure 3. Normalized absorption spectra show the spread of the impurity band as the impurity concentration is increased. 